$(\mathrm{n}=4)$, Thailand $(\mathrm{n}=3)$, Australia $(\mathrm{n}=1)$, Russia $(\mathrm{n}=1)$, Sweden $(n=1)$ and Malaysia $(n=1)$. Therein, $84 \%$ sequences mixed among CN.01AE.HST/IDU-2, most of which were sampled before 2010 and born in Southeast Asia. 14\% sequences clustered with CN.01AE.MSM-4 and -5. A distinct sub-cluster with small divergence that consisted of 15 Japanese variants was formed inside CN.01AE.MSM-4 and the tMRCA of CN.01AE.MSM-4 and the latter sub-cluster were around 1996 and 2006. For other four major lineages, we have not found any significant transmission cluster structure for specific geographical regions.

Conclusion This results demonstrated the Chinese CRF01_AE variants have disseminated to other countries but have not scaled, and the immigrant modes of CRF01_AE variants from Asia to global has changed. Our study provided the evidences of regional and global dissemination of Chinese CRF01-AE lineages and highlighted the importance of shaping the newest global landscape of HIV and the necessity to implement the effective measures to reduce global spread of HIV.

Disclosure No significant relationships.

\section{P149 EVOLUTION: A TEXT MESSAGING POWERED INTERVENTION FOR CONNECTION, SUPPORT AND HIV ERADICATION}

${ }^{1}$ Katie Plax*, 'Jeff Glotfelty, ' $J u l i a$ Schlueter, ${ }^{2}$ Donny Gerke, ${ }^{1}$ Stacey Slovacek, ${ }^{1}$ Maria Freshman, 'Kim Donica. 'Washington University School of Medicine, Pediatrics, St Louis, USA; ${ }^{2}$ University Of Denver, Graduate School of Social Work, Denver, USA

\subsection{6/sextrans-2019-sti.314}

Background To reach the UNAIDS goal of 90-90-90, young people worldwide must experience improved linkage to care, retention in care and viral load suppression (VLS). This goal highlights needs for accessible mHealth approaches. The EVolution intervention is one such approach with automated and live text-messaging and a triggered alert system for timely support. Our goals included: 1) address concerns in young people's lives (housing, utilities, and mood); 2) increase appointments kept; 3) improve VLS rates.

Methods HIV postive youth at clinic sites with access to a mobile texting device, and at least one additional criterion: newly diagnosed; not linked to care; out of care for at least six months; viral load greater than 200 copies $/ \mathrm{mL}$ were recruited. The intervention uses automated two-way text messages for HIV-specifc medication/appointment reminders, monthly housing/utilities needs assessment and semi-weekly mood checks. Self-reported client challenges trigger action alerts and timely live two-way text messging with case management. Results were examined over 6-months.

Results 102 youth enrolled. 89 participated in the program for $\geq 6$ months. Most participants were young, average age $22.33(\mathrm{SD}=2.08)$, male $(91 \%)$, Black $(93 \%)$ and men who have sex with men (MSM) (82\%). Eighty-one percent of youth responded to at least one text in 89,120 total exchanges. Texts triggered 395 alerts, most often for missed medication, housing issues and missed appointments. Sevetynine youth kept at least one appointment for HIV medical care. Twenty-five participants newly achieved VLS and 35 maintained VLS during the 6 months. A significantly greater proportion of participants were virally suppressed at 6 months versus baseline $(p=0.18)$

Conclusion This text messaging intervention, which could be replicated in diverse settings, led to improved health and communication in a sample of young people at-risk or experiencing poor HIV outcomes. This presentation introduces an innovative streamlined communication approach with HIV postive youth, which addresses important health and social needs.

Disclosure No significant relationships.

\section{P151 HIV EPIDEMIC AMONG CRACK USERS IN CENTRAL BRAZIL: EPIDEMIOLOGICAL BASIS FOR INTERVENTIONS}

${ }^{1}$ Karlla Caetano*, ${ }^{2}$ Sheila Teles, ${ }^{3}$ Ana Rita Motta-Castro, ${ }^{2}$ Megmar Carneiro, ${ }^{4}$ Divânia França, ${ }^{4}$ Leandro Silva, ${ }^{5}$ Viviane Castro, ${ }^{1}$ Márcia Souza, ${ }^{4}$ Grécia Pessoni. ${ }^{1}$ Universidade Federal de Goias, Faculty of Nursing, Goiânia, Brazil; ${ }^{2}$ Universidade Federal de Goias, Goiânia, Brazil; ${ }^{3}$ Universidade Federal de Mato Grosso, Campo Grande, Brazil; ${ }^{4}$ Municipal Health Department of Goiânia, Goiânia, Brazil; ${ }^{5}$ University Hospital Maria Aparecida Pedrossian, Campo Grande, Brazil

\subsection{6/sextrans-2019-sti.315}

Background Global strategies have been discussed and implemented worldwide to reduce the number of new HIV infections among key populations. In Brazil, there is a large lack of knowledge about groups that are highly vulnerable to this infection, such as drug users. Therefore, this study aimed to investigate the epidemiological profile of HIV infection in institutionalized crack users in the Central Region of Brazil.

Methods Between 2012 and 2013 we conducted a cross-sectional study of 919 crack users who were being treated at mental health referral institutions in two major cities located in the Central-West Region of Brazil. All were interviewed and tested for anti-HIV by the enzyme-linked immunosorbent assay (ELISA). Univariate and multivariate Logistic regression were done to identify the association of HIV and behaviors variables. This study was approved by the Research Ethics Committee of the Federal University of Goiás.

Results Of the total number of individuals recruited, $85.2 \%$ were male $(84.5 \%)$, young (median: 30 years old, IQR: 1868) and basic schooling (median: 8 years of study, IQR: 018). Of the total, $2.6 \%$ (95\% CI: $1.7 \%-3.8 \%$ ) were anti-HIV positive, ranging from $2.2 \%$ (95\% CI: $1.4 \%-3.6 \%)$ in men and 4.4\% (95\% CI: 2\%- 9.2\%) in women. Logistic regression analysis revealed that the antecedent of life on the street (adjusted OR: 4.0, $p=0.023$ ), sexually transmitted infection (adjusted OR: 4.4, $p=0.014$ ), use of improvised pipe for crack use (adjusted OR: $7.9 ; p=0.010$ ) and having sex in the last year with a partner known to have HIV/AIDS (adjusted OR: $8.7 ; p=0.001$ ) were predictors of HIV infection.

Conclusion Effective HIV prevention and control strategies should be implemented for this specific group including the community-based and community-led programs, with an emphasis on health education, harm reduction measures, and control of sexually transmitted infections, especially among homeless people.

Disclosure No significant relationships. 